\title{
Italian Risorgimento and the European Volunteers
}

\author{
Antonello Battaglia
}

Sapienza University of Rome, Italy

\author{
Doi:10.5901/ajis/2013.v2n1p87
}

\section{Abstract}

The Risorgimento was a complex phenomenon, not only an Italian one, that had broad resonance throughout Europe since the "rise of non-historical nations" spread particularly in central and eastern part of the continent. In 1815 the Congress of Vienna worked to restore the status quo in order to set aside the Napoleonic experience. The anachronism of the measures was already highlighted just five years later when many uprisings erupted in Europe, and especially with the "national" revolutions of 1848-1849, when Poles, Hungarians, Croats, Serbs, Czechs, Italians, Bulgarians shared the principles of freedom and joined the slogan: "For your freedom and ours" was the slogan that brawny European volunteers in the continental Risorgimento confirming an irreversible crisis of the multinational empires.

Key Words: Volunteers, Insurrections, Risorgimento, Garibaldini, History of Europe.

Il Risorgimento, complesso e variegato, non fu soltanto un fenomeno italiano ma ebbe vasta risonanza nello scacchiere continentale che vide il "risveglio delle nazionalità non storiche" diffondersi in particolare nell'Europa centro-orientale.

Il Congresso di Vienna aveva ripristinato lo status quo antecedente le guerre napoleoniche e la diplomazia europea aveva condotto una restaurazione geopolitica sul solco delle tradizioni settecentesche. L'anacronismo dei provvedimenti venne messo in luce già appena cinque anni dopo, quando i primi moti scoppiati in Spagna nel I820-I82I rivelarono il diffuso malcontento soffocato prontamente sul nascere anche a Napoli e in Sicilia. Dieci anni più tardi le trois glorieuses rovesciarono nuovamente i Borbone in Francia e inaugurarono la breve parentesi di Luigi Filippo d'Orleans mentre Belgio e Grecia raggiungevano l'agognata indipendenza rispettivamente da Olanda e Impero Ottomano e a Varsavia e Modena fallivano i moti.

La crisi del 1848 ebbe maggiore risonanza e inedita forza d'urto che travolsero l'intero continente rilevando definitivamente l'obsolescenza dell'ordine geopolitico e la necessità di costituirne uno nuovo (Biagini 201 I).

La cosiddetta primavera dei popoli - che quindi non fu meramente confinata alla penisola italiana - si diffuse per osmosi in molte aree. Il Risorgimento accomunava così gli italiani ai polacchi che dal I795 - anno della terza spartizione - si opponevano alle potenze "partitrici"; ai croati, animati dall' "Illirismo" di Gaj; ai magiari la cui causa era perorata dall' attività insonne degli esuli politici; ai romeni in questo frangente aspiranti all'autonomia; ai serbi, sempre più coscienti del progetto di divenire il popolo guida degli slavi meridionali; ai greci, già protagonisti della propria indipendenza nel $1830 \mathrm{ma}$ decisi a scalzare definitivamente il controllo ottomano e infine anche i bulgari agli albori dello sviluppo della propria coscienza nazionale (Tamborra I983). I grandi imperi multinazionali, asburgico e ottomano, da secoli 
garanti della stabilità geopolitica in Europa centro-orientale vennero messi seriamente in crisi dalle forze centrifughe.

I volontari costituirono la fondamentale forza propulsiva del movimento rivoluzionario e non sarebbe corretto pensare al Risorgimento senza valutare adeguatamente il loro determinante contributo nella difesa degli ideali di libertà e unità nazionali. Dopo le lotte armate in Uruguay contro l'esercito di Rosas, governatore generale della provincia di Buenos Aires, Garibaldi tornò sulla scena europea sbarcando a Nizza il 2I giugno I848 con sessantatrè uomini (fra cui Medici, Sacchi e Anzani).

\section{"L'America che nel I836 aveva raccolto un oscuro marinaio e un disertore proscritto restituiva all'Italia un ammiraglio provetto, un capitano invitto, un eroe glorioso".(Guerzoni 1982).}

Come scrive Tamborra «In Europa - e soprattutto in Italia - le ripercussioni delle sue gesta e la sua ascesa nella scala dei valori e politici, sono tali che già negli ambienti sotterranei dei cospiratori - vi è una "attesa" per quello che un simile uomo potrà compiere a favore della sua Patria e, quindi, anche di "altre" patrie» (Tamborra 1983).

In occasione della Prima Guerra d'Indipendenza, il patriota polacco Adam Mickievicz, il 3 maggio 1848, chiese al Governo provvisorio di Lombardia la creazione di una Legione polacca in cui avrebbero dovuto arruolarsi cechi, croati e serbi disertori dell'esercito austriaco oltre a emigrati polacchi. Le compagnie costituite furono due, entrambe sotto il comando di Kamieński e furono impegnate nei combattimenti ai confini del Tirolo, una alle dipendenze delle truppe comandate dal generale Durando e l'altra, sotto l'egida di Siodłkowicz, partecipò alla difesa di Milano. Dopo i primi scontri le legioni vennero spostate in Piemonte e una parte di volontari, le cui fila vennero rimpinguate da ulteriori rinforzi provenienti dalla Sicilia e guidati da Chodzko, giunsero a Livorno dove combatterono valorosamente. Il 10 ottobre 1848, Chodzko concluse con il Governo provvisorio toscano di Guerrazzi e Montanelli un accordo secondo il quale metteva per due anni a disposizione dei repubblicani 800 volontari, liberi di tornate in Polonia qualora fosse scoppiata una rivoluzione nazionale. Mickiewicz contestualmente riuscì a riunire un gruppo di 150 volontari polacchi che partì sotto il comando del capitano Alessandro Fijalkowski per Genova e prese parte attiva alla sommossa della città. Successivamente imbarcata per Livorno e Firenze, ormai in mano alla repressione, la legione raggiunse il litorale laziale e si mise a disposizione della Repubblica Romana. Il triumvirato costituito da Mazzini, Saffi e Armellini, riconobbe ufficialmente la legione con decreto datato 29 maggio I849. In questa occasione venne aggiunto il nastro tricolore allo stendardo e ancora una volta venne ribadito il diritto di ritornare in patria in caso di insurrezione.

«Per la vostra libertà e la nostra» era il vessillo polacco innalzato e sventolato dalla legione del colonnello Milbitz che combatté il 3 giugno a Villa Pamphili e il I5 giugno a Ponte Milvio contro le truppe francesi. Dopo la caduta della Repubblica, i polacchi si arresero al generale Oudinot che decise di imbarcali e confinarli a Corfù.

Oltre che a Roma, gli artiglieri polacchi si distinsero a Venezia, nella difesa della Repubblica e a Morazzone dove il capitano Pawel Bielski e I3 compagni si batterono nella 
battaglia coadiuvando Garibaldi contro gli austriaci ${ }^{1}$. Nel 1849 al generale Chrzanowski venne affidato il comando dell'esercito sardo contro le forze di Radetzki che avevano ripreso il controllo dell'area lombarda. Al sud, in Sicilia, le forze rivoluzionarie vennero poste sotto l'egida del generale Mierosławski, appena reduce dalla sfortunata insurrezione della Posnania.

La crisi del I848-'49 rappresentò dunque il primo punto di contatto e di convergenza fra la nazionalità italiana e quelle dell'Europa centro-orientale, il Piemonte era strettamente vicino ai destini dei popoli e il fallimento dei moti rivoluzionari non annichilì tuttavia tale legame. Il regno di Sardegna - sotto il neo sovrano Vittorio Emanuele II e l'abile attività politica del primo ministro Cavour - divenne il baricentro delle aspirazioni dei popoli "in via di definizione” (Salvatorelli I957). L'intervento piemontese nella “crisi d'Oriente” del I853-'56 fu l'occasione sfruttata da Cavour per proiettare e inserire il proprio paese nello scacchiere diplomatico internazionale. Per la prima volta, infatti, al congresso di Parigi venne sollevato il problema italiano. L'intesa del Regno di Sardegna con il Secondo Impero di Napoleone III che con il suo messianesimo si ergeva a nuovo liberatore dei popoli - venne salutata dalle popolazioni centro-orientali come una concreta possibilità di indebolimento del potere austriaco e auspicio per una rivoluzione generalizzata. I contatti di Garibaldi, Mazzini e Cavour con i movimenti rivoluzionari clandestini ne sono la testimonianza. Come noto, gli accordi di Plombières, siglati il 2I luglio I858, portarono alla Seconda guerra d'Indipendenza e alle reali possibilità di una conquista del Lombardo-Veneto, mentre gli insorti polacchi, di cui molti emigrati da una Polonia di fatto spartita e inesistente, e ungheresi guidati rispettivamente da Czartoryski e Kossuth speravano in una distrazione militare austriaca che potesse permettere di insorgere e ottenere agevolmente l'indipendenza. L'armistizio di Villafranca, siglato l'I I luglio I859, fu una grande delusione non solo per Cavour, che per protesta si dimise, ma per tutte le nazionalità insorgenti che videro sfumare nuovamente la possibilità di un aiuto proveniente da una delle grandi potenze continentali. Come rileva Biagini (20II), si trattò di un momento di disillusione e costernazione in cui i popoli dell'Europa centro-orientale pensarono di essere stati definitivamente abbandonati al potere dei grandi Imperi multinazionali (Tamborra 1958).

Appena un anno dopo, il successo dell'impresa dei Mille e la proclamazione dell'Unità italiana risvegliarono il fervore nazionale.

Il 5 maggio I860, come noto, Garibaldi con i due piroscafi Lombardo e Piemonte salpò dallo scoglio di Quarto e giunse a Marsala giorno II. Dopo il sanguinoso scontro di Calatafimi e l'entrata in Palermo, si fece appello ai volontari europei che accorsero in Sicilia. I nuovi garibaldini affluirono a Genova, vennero inquadrati nelle divisioni Medici e Cosenz in attesa di imbarcarsi per la Sicilia a bordo dei piroscafi Washington, Oregon e Franklin. Numerosi furono i triestini, gli istriani e i dalmati «appartenenti alle ultime propaggini della nazione italiana, ad una italianità marginale posta cioè agli estremi limiti della espansione territoriale della italianità, là dove viene a contatto e contrasto con altre stirpi e altre lingue e illanguidisce, e muore» (Sestan 1947) La loro rinnovata presenza tra le truppe garibaldine aveva un significato profondo: attestazione di volontà nazionale, coscienza di un legame,

\footnotetext{
${ }^{\text {I }}$ Il generale nizzardo ne parla nelle sue Memorie.
} 
esistenza di una spinta interiore che li rendeva partecipi della vicenda unitaria della patria comune. In particolar modo i garibaldini dalmati si erano già distinti nella difesa di Venezia. Gli zaratini Giuseppe Zmaich², Giovanni Maggiorato, Carlo Tivaroni, Giacomo de Zanchi, Costantino Venturini, Doimo de Hoebert, Spiridione Galateo, Enrico Matcovich di Stretto, De Giovannizio di Spalato; Lorenzo Girotto di Spalato, Eugenio Popovich, il colonnello Cossovich, i due fratelli Millanovich, Spirione Sirovich, Lisovich di Budua, Luigi e Federico Seismit Doda, alcuni di loro avrebbero combattuto a fianco di Garibaldi in Trentino nel I866, a Mentana nel I867 e in Francia nel 1870-'7I (Cace 186I). Altri triestini e istriani si erano già distinti nella difesa di Roma: Giacomo Venezian era stato ucciso a Porta S. Pancrazio, erano stati feriti anche Zucchi e Zambeccari, mentre Zamboni, Bruffel erano stati colpiti a Porta Cavalleggeri dove avevano combattuto anche Sansoine Levi, Salvi, Marocchino, Hoenigmann, Sanzin, Mitis di Cherso, Baccalari di Dignano d'Istria, promosso maggiore proprio durante la difesa della Repubblica Romana. Nel 1859 le fila dei "Cacciatori delle Alpi” erano state rafforzate proprio da istriani e dalmati e soprattutto l'impresa dei Mille e le successive spedizioni videro un afflusso notevole di volontari anche triestini e giuliani. Due dei Mille sono conosciuti: Cesare Michieli di Campolongo che combatté a Calatafimi, a Palermo, a Milazzo e sul Volturno. Marziano Giotti di Grandisca, della compagnia di Cairoli, decorato al valor militare. Inoltre il capitano mercantile Enrico Maffei, triestino, Giovanni Bertossi, decorato al valore sul Volturno, Gustavo Büchel, Gioacchino Sibel, Daniele Wertheimer, Arminio Wurmbrand, Federico Cuder di Capodistria; tre di Rovigno si distinsero in modo particolare: Luigi Dasarsa, Giorgio Moscarda, Baldassarre Manzoni, Enrico Appel (Sticotti 1932). Tra i Mille si sono da annoverare anche vari ebrei tra cui Ciro Finzi, Cesare de Enrico Guastalla, i fratelli Alessandro, Isacco, Israele Levi, il tedesco Adolph Moses, Antonio Alpromi, Antonio Godberg, Riccardo Luzzatti, Eugenio Rava, Guido Rovighi, Davide ed Enrico Uziel e Giovanni Acerbi ${ }^{3}$. Va segnalata inoltre, tra le fila dei garibaldini nella stagione 1860-'6I, la presenza di numerosi italo-albanesi. Il passaggio di Garibaldi in Calabria e il proclama del generale al vigore dei "discendenti di Skanderbeg" favorirono la costituzione di un reggimento italo-albanese al comando del colonnello Domenico Damis di Lungro tra cui un gruppo di giovani scappati dal collegio di Sant'Adriano (Pacukaj 20I I). Tra i volontari che si batterono ai ponti della Valle e al Volturno i più noti furono Raffaele e Domenico Mauro di San Demetrio Corone, Giuseppe Pace di Castrovillari, il maggiore Gennaro Placco di Civita, già compagno di Luigi Settembrini nel penitenziario di Procida, Gennaro Mortati di Spezzano Albanese (Groppa 1912).

"Gli albanesi sono eroi che si sono distinti in tutte le lotte contro la tirannide» affermò il 2 ottobre Garibaldi mentre donava 12000 ducati al collegio di S. Adriano a S. Demetrio Corone (Lorecchio 1904).

Notevole l'afflusso dei volontari francesi, soprattutto emigrati che protestavano delusi dalla politica di Napoleone III. Difficile definirne il numero preciso e l'orientamento politico.

\footnotetext{
${ }^{2}$ Archivio di Stato, Mantova, Carte Acerbi (cit. da Tamborra).

${ }^{3}$ G. De Angelis, Garibaldi romanziere de «I mille» e gli ebrei in « La Rassegna mensile di Israele», vol. XXV, n. I I, pp. 453 e sgg. I fratelli Uziel sono ricordati da Garibaldi ne I Mille, vol.II, pp.88, 9I, 345.
} 
Dagli studi di Ferdinand Boyer si individuano 59 garibaldini francesi, mentre tenendo in considerazione ulteriori scaglioni partiti successivamente per il meridione italiano, Tamborra sostiene che il numero si aggirasse tra i 300 e i 500. Tra la fine di giugno o i primi di luglio, a Parigi venne costituita la Mission Sicilienne, di cui fece parte anche il medico Leullier, anch'egli inviato successivamente nell'Isola.

Il gruppo più importante di volontari transalpini fu la "Legione" comandata dal visconte Paul de Flotte, esule dopo il colpo di Stato napoleonico del 2 dicembre I85I. Fu il garibaldino francese di maggior rilievo che con la sua unità - circa 250 effettivi inquadrati nella $15^{\text {ma }}$ divisione Türr - combatté a Solano e morì in battaglia il 22 agosto I860, durante lo sbarco in Calabria (Motta 201I). In suo onore Garibaldi diede il nome "Compagnia La Flotte" 4 all'unità dei francesi. Un'altra figura di rilievo fu quella di Philippe Toussaint Joseph Bourdon, comunemente conosciuto come Bordone. Ricevette da Garibaldi l'incarico di dirigere la fonderia di cannoni in bronzo di Palermo. Da Milazzo in poi divenne membro dello Stato Maggiore di Garibaldi, distinguendosi per le sue qualità di sovrintendente ai materiali d'artiglieria. Nella battaglia del Volturno schierò nuove batterie, costruì un ponte che permise a numerosi volontari di passare sull'altra sponda. Nella guerra franco-prussiana avrebbe seguito Garibaldi nell'“Armata dei Vosgi” (Boyer 197I)

Di grande importanza fu anche la figura di Maxime du Camp, scrittore già noto, che giunse in Sicilia come ufficiale di Stato Maggiore della divisione Türr e accompagnò Garibaldi fino a Napoli. I suoi ricordi, affidati alla autorevole Revue de deux mondes, furono riuniti in un volume dal titolo Expedition de deux Siciles (Parigi, I88I) e da lui ripubblicate nei suoi Souvenirs Littéraires da cui si cita:

«Pendant quatre mois passés à l'état-major du général Turr où les éléments italiens, anglais, hongrois et francais étaient mêlés dans d'inégales proportions, je n'ai pas assisté à une seule dispute; je n'ai pas intendu un mot plus vif qu'il n'aurait convenu» (Camp I86I).

Altro personaggio francese rilevante fu lo scrittore Alexandre Dumas (padre) che partì con la propria goletta in crociera per il Mediterraneo e non appena ricevette la notizia delle sommosse siciliane, fece rotta per l'Isola. Rimasto sulla sua nave ancorata nel porto di Palermo, fece visita a Carlo Pellion di Persano, (I869) comandante della squadra navale sarda, a cui confidò di voler finanziare i volontari garibaldini. Pochi giorni dopo acquistò a Marsala 550 carabine e 10.000 cartucce. Dopo il passaggio dello Stretto da parte di Garibaldi, lo scrittore transalpino si recò con la sua goletta Emma a Salerno per preparare e avviare una massiccia propaganda anti-borbonica. Grazie ai propri informatori, entrò in contatto con il ministro dell'Interno e di Polizia del governo costituzionale, Liborio Romano e con lo zio di Francesco II, il conte di Siracusa, uomo liberale, contribuendo in maniera trionfale all'ingresso di Garibaldi a Napoli a cui non assistette perché, venuto a conoscenza dell'imminente insurrezione ad Avellino, decise di dirigersi verso sud, a Messina dove imbarcò nuove armi per

${ }^{4}$ F. Boyer, Les volontaires français avec Garibaldi en I860, in «Revue d'Histoire moderne et contemporaine», aprile-giugno 1860, pp. I26-I27. A. Colocci, Paul de Flotte, Bocca, Torino 1912. C. Pecorini Manzoni, Storia I5ma Turr nella campagna del 1860 in Sicilia e Napoli, Gazzetta d'Italia, Firenze I876, pp. XI, I35, 290. 
trasportarle in Campania. L'incontro con il generale nizzardo avvenne poche settimane dopo e in quest'occasione i due, salutandosi, si abbracciano e Dumas così scrisse:

«Ah! Te voilà, s'écriait-t-il en me voyant. Dieu merci, tu t'ss fait assez attendre! C'était la première fois que le général me tutoyait. Je me jetai dans ses bras en pleurant de joie» (Boyer 1960)

Sulla scia di Dumas, si mossero anche altri illustri scrittori come Victor Hugo, George Sand, Edgar Quinet etc. I francesi provenivano da molti strati sociali: politici, intellettuali, nobili, popolani, professionisti, contadini, ex soldati e operai di diverse zone: Parigi, Provenza, Bretagna, Corsica, Nizza e Savoia.

Per quanto riguarda gli inglesi, l'impresa dei Mille ebbe vasta risonanza presso l'opinione pubblica e l'afflusso di volontari fu immediato e alquanto cospicuo ${ }^{5}$. La legione inglese infatti era costituita da 456 unità di cui 24 ufficiali al comando maggiore Carlo S. Smeld. Anche il brigadiere Giovanni Dunne si imbarcò per la Sicilia e arruolò nel suo reggimento - con l'appoggio del tenente colonnello Percy Wyndhan e dei connazionali Patterson e Dowling, molti siciliani che costituirono una nuova unità. John Whitehead Peard e Hugh Forbes, chiamati "the Garibaldi’s english men", vennero inquadrati nella II compagnia Pavia costituita quasi esclusivamente da studenti che avevano in dotazione revolver rifles ${ }^{6}$, nuova arma inviata dall'inglese Colt ai garibaldini. I due, distintisi entrambi in battaglia, si divisero a Milazzo dove Hugh, con enorme rammarico di Garibaldi, lasciò la formazione accettando la carica di governatore della città mentre il collega, alla guida di 1000 volontari britannici, seguì il generale fino alla battaglia del Volturno. Altro importante contributo fu quello delle testate giornalistiche come l'«Independent» di Jersey e il «London Trades Council» che promossero la sottoscrizione per l'invio di volontari in Sicilia (Valiani I960).

Tra i garibaldini stranieri inquadrati nella $15^{\mathrm{ma}}$ divisione Türr, (Fornaro I996) brigata Eber, si ricordano anche gli elvetici Luigi de Niederhausern, maggiore, Carlo d'Almen, capitano (Camp I88I) e il prussiano Wihlelm Rüstow, colonnello e successivamente capo di Stato Maggiore della stessa divisione.

Notevole, dunque, fu la presenza di volontari provenienti dall'Europa occidentale ma ancora più massiccio fu l'afflusso di uomini dalle propaggini centro-orientali del continente. Oltre ai polacchi, ai quali ho già accennato in precedenza, furono gli ungheresi i più numerosi. Primo fra tutti, il generale Stefano Türr, a capo della $15^{\text {ma }}$ divisione e molti magiari nella divisione Medici. Dopo la presa di Palermo, venne costituita una legione ungherese che raggiunse il numero di 440 unità a cui si aggiunsero gli ussari magiari già in servizio nell'esercito borbonico. Tra loro si ricordano i comandanti: F. Eber, K. Eberhardt e F. Pulszky,

\footnotetext{
${ }^{5}$ AA. VV., Scritti e discorsi politici e militari, Vol. I, Cappelli, Bologna, 1934, pp. 274-275,337, Risposta di Garibaldi a un indirizzo giuntogli da Sheffield, Palermo, I3 luglio I860; analog. al Comitato di Glasgow, Caprera, 30 novembre I860.

${ }^{6}$ Archivio del Risorgimento, Roma, Busta 45, fasc.27. Traduzione dell'epoca del Rapporto del Colonnello Forbes intorno agli affari della legione inglese venuti sotto la sua immediata attenzione, inviato a Napoli al Comitato di Londra il 28 novembre 1860 .
} 
i comandanti di brigata Gustavo Frigyesy e Szakmary, i tenenti colonnelli R. Magyoródy che si distinse al Volturno e F. Figylmesy, M. Kiss, A. Teleki e infine L. Winkler, comandante del $4^{\circ}$ reggimento, brigata Sacchi, della I $^{\text {ma }}$ divisione Türr (Türr 1928). Da non dimenticare anche Lajos Tüköry che partecipò alla battaglia di Palermo come comandante dell'avanguardia che superò per prima le linee nemiche. Proprio durante lo scontro del 27 maggio venne ferito a una gamba che si infettò e, nonostante venisse amputata, lo portò alla morte. In suo onore Garibaldi ribattezzò l'ex corvetta a ruote partenopea Monarca, proprio Tüköry (Colombo 1932).

"Nell'insieme, una partecipazione massiccia, questa degli ungheresi che dall'Italia guardano come meta all'Ungheria, ben consapevoli di poter contare sulle parole dette da Garibaldi il I6 ottobre I860: "Ad essi non solo dobbiamo gratitudine, ma è nostro dovere aiutare la loro causa e farla nostra... La libertà d'Italia è strettamente legata all'indipendenza e alla libertà d'Ungheria"» (Kastner 1960).

Anche i boemi parteciparono intensamente all'attività rivoluzionarie. A seguito della loro attività molti furono costretti a diventare esuli: Josef Václav Frič, Eduard Rüffer, Jindřich Podlipský, Karel Výma di Letohrad, Rudolf Gasparovský di Pardubice, Jan Macháče, Jan Steinbachk. I greci, testimoni di un antico legame con la penisola italiana - per i molti volontari italiani che avevano parte all'insurrezione greca del I82I - accorsero numerosi agli ordini di Garibaldi: primo fra tutti Santorre di Santarosa e altri ancora, Stamatis Tipaldos, Dionisos Mavropulos e Sotirou Zisis. Elias Stecoulis salpò con i Mille da Quarto e seguì Zambianchi nella diversione sulla costa maremmana, partecipò allo scontro di Grotte di Castro contro i soldati pontifici dove venne catturato e in seguito rilasciato insieme ad altri connazionali (Kerofilas I919).

Quanto ai bulgari, si distinsero Vasil Levski, Georgi Sava Rakovski, lo scrittore e poeta Ljuben Karavelov, Marko Balabanov, Hristo Botev. «Per tutti il binomio Mazzini-Garibaldi entra a far parte del patrimonio comune dell' intellighenzia rivoluzionaria bulgara». Il primo garibaldino bulgaro fu Dmitar Pehlivanov, pittore, giunto a Roma per perfezionare gli studi e in seguito arruolatosi nelle fila dei volontari garibaldini alla difesa della Repubblica Romana con il compatriota Georgi Kapčev;

Gjuro Načev fece invece parte dei "Cacciatori delle Alpi” e nel I866 tornò in Italia con gli amici Neno Marinov e Ivan Hagidimitrov, detto "garibaldito” (Petkanov I966). Un importante volontario romeno-bulgaro fu Stefan Dunjov, che si batté insieme alla legione ungherese e venne ferito nel I860 durante la battaglia del Volturno per la quale, tra l'altro, venne insignito di medaglia d'argento al valor militare ${ }^{7}$. Anche i volontari russi accorsero numerosi alla chiamata di Garibaldi. I figli di un non ben identificato Bernov scapparono di casa per raggiungere il generale a Roma. Lo scrittore G. Korolenko racconta di un garibaldino ucraino, un certo Massimo, originario della Volinia, arruolatosi tra i volontari per il suo odio

\footnotetext{
${ }^{7}$ La grave ferita comportò l'amputazione di una gamba per la quale il Governo italiano corrispose una pensione di guerra.
} 
nei confronti dell'Austria e tornato in Russia, dopo molti anni, come "invalido garibaldino"; non mancò una donna russa, originaria di Mosca, moglie di un garibaldino. Altra famosa figura storica fu quella della contessa Nesselrode, più nota con lo pseudonimo "principessa Drousckoy”, che arruolò i garibaldini e a Firenze si prodigò per la propaganda rivolta ad attrarre nuovi giovani. Uno dei più noti fu Lev Il'ič Mečnikov di Pietroburgo, che passò da Milano a Venezia ma non riuscì a raggiungere in tempo Quarto per riunirsi ai Mille. Entrò comunque a far parte della formazione comandata dal colonnello Giuseppe Nicotera, sbarcò a Napoli il 7 settembre 1860 contestualmente all'arrivo di Garibaldi dal sud. Non giunse invece in tempo l'anziano colonnello Ditmar che arrivò dopo la conclusione della campagna garibaldina (Venturi 1960).

Complessivamente, il numero di volontari stranieri fu rilevante, ammontò a circa 2500 combattenti che, insieme agli italiani, coadiuvarono Garibaldi soprattutto nell'impresa meridionale.

Il garibaldino divenne l'esempio da emulare per tutti i popoli e Garibaldi l'eroe internazionale in grado di dare aiuto, con le sue milizie, ai risorgimenti dell'est. Dopo le delusioni del I849, l'Unità italiana fu vista dalle popolazioni dell'Europa centro-orientali come il primo grande successo di una rivoluzione. Nell'immaginario comune la camicia rossa (Motta 20II) divenne la divisa che univa uomini di varia origine, di diversa estrazione nazionale uniti a Garibaldi per un ideale comune, quello della libertà e dell'indipendenza nazionale.

Cesare Balbo in Speranze d Italia (I844) - inseguendo un'idea che sembrava utopistica nel ritenere che l'Unificazione italiana infiammasse tutti i popoli "da riscattare" - fu forse il primo a mettere in relazione il Risorgimento italiano con l'area centro-orientale europea (Becherelli 20I I). Nel I86I, dopo circa un ventennio, l'idea divenne plausibile e concreta agli occhi delle diplomazie europee. La "questione italiana" si era ormai legata indissolubilmente a quella delle nazionalità e contribuiva a favorire le spinte nazionali degli altri risorgimenti forzando gli schemi di un'Europa che doveva cambiare. La partecipazione corale di ungheresi, polacchi, russi, albanesi, greci, romeni, dalmati, istriani, francesi e inglesi, mostra che il Risorgimento non fu solo un fenomeno italiano, ma si espanse e coinvolse tutti gli europei che si riconobbero in un progetto comune di libertà e democrazia in sintonia con un'idea condivisa di identità europea (Biagini \& Carteny 2010).

\section{Bibliografia}

M. Cace, (I86I), I patrioti dalmati e l'unità d'Italia, in «Il Messaggero Veneto», I6 marzo I86I;

C. Pellion di Persano, (I869), Diario privato-politico-militare nella campagna navale I860 e I86I, Civelli, Firenze.

C. Pecorini Manzoni, (I876), Storia I5ma Türr nella campagna del 1860 in Sicilia e Napoli, Gazzetta d'Italia, Firenze.

M. du Camp, I88I), Expédition des Deux Siciles, Calmann Levy, Parigi I88I;

A. Colocci, Paul de Flotte, Bocca, Torino I9I2;

S. Groppa, Gl'italo-albanesi nelle lotte dell'indipendenza, Lella e Casini, Bari I9I2;

C. Kerofilas, La Grecia e l'italia nel Risorgimento italiano, Libreria della Voce, Firenze I9I9;

S. Türr, L'opera di Stefano Türr nel Risorgimento italiano, Tipografia Fascista, Firenze I928;

A. Lewak, Corrispondenza polacca di G. Garibaldi, Cracovia, I932;

P. Sticotti, La Regione Giulia nelle guerre per l'indipendenza, Società editrice mutilati e combattenti, Trieste I932; 
A. Colombo, Il generale de Milbitz nel Risorgimento italiano, in Atti del Congresso storico internazionale di Varsavia, I933;

AA. VV., Scritti e discorsi politici e militari, Vol. I; Cappelli, Bologna, I934;

E. Sestan, Venezia Giulia. Lineamenti di Storia etnica e culturale, Edizioni Italiane, Roma I947;

L. Salvatorelli, La politica estera, in L'Italia dal I86I al I870, Atti del X Convegno Storico Toscano, Cortona, 25 28 aprile 1957, in «Rassegna storia toscana», anno III, fasc. III-IV, luglio-dicembre I957;

A. Tamborra, Cavour e i Balcani, Ilte, Torino, I958;

F. Boyer, "Les Garibaldiens” d'Alexandre Dumas: roman ou choses vue, in «Studi Francesi», n.I0, Torino, I960;

F. Boyer, Les volontaires français avec Garibaldi en I860, in «Revue d'Histoire moderne et contemporaine», aprilegiugno I960;

K. Kastner, Kossut-emigráció olaszországban, (L’emigrazione di Kossuth in Italia), Budapest I960;

L. Valiani in «Atti del XIII Convegno Storico Toscano. Porto Santo Stefano 29 maggio-I ${ }^{\circ}$ giugno I960», pubbl. in «Rassegna Storica Toscana», fasc. IV, I960;

F. Venturi, L’immagine di Garibaldi In Russia all'epoca della liberazione dei servi, in «Rassegna storia toscana», ottobre-dicembre I960;

G. Falzone, Lettere di Garibaldi a Elia Stekuli, in «Il Risorgimento», Milano I965;

I. Petkanov, Riflessi del Riflessi del Risorgimento in Bulgaria, in «Rassegna Storica del Risorgimento», I966;

F. Boyer, Un garibaldien français: le général Bordone in «Rassegna Storica del Risorgimento», I97I; G. Guerzoni, Garibaldi, Firenze 1982;

A. Tamborra, Garibaldi e l'Europa. Impegno militare e prospettive politiche, Fusa, Roma I983;

P. Fornaro, Risorgimento italiano e questione ungherese (I849-I847), Rubbettino, Soveria Mannelli, I996;

P. J. Proudhon, Contro l'Unità d'Italia, Miraggi, Torino, (2010), a cura di A. Biagini, A. Carteny;

G. Motta, Baroni in camicia rossa, Firenze , Passigli 20II;

S. Pacukaj (20II) Il risorgimento italiano e gli arberesh in Ripensare il Risorgimento a cura di G. Motta, Nuova Cultura, Roma.

G. Motta (a cura di), Ripensare il Risorgimento, Nuova Cultura, Roma, 201 I. 
